(Aus der Medizinischen Klinik der Universität Königsberg.)

\title{
Uber die Wirkung \\ von Gefäßmitteln auf den Venendruck des Menschen.
}

\author{
1. Mitteilung. \\ Adrenalin, Papaverin, Strychnin.
}

Von

Privatdozent Dr. Georg Rosenow.

Mit 4 Textabbildungen.

(Eingegangen am 1\%. Oktober 1919.)

Die Kenntnis der Wirkungsweise der Vasomotorenmittel auf den Kreislauf des Menschen entstammt, wie die der meisten Arzneikörper zwei Quellen, dem Experiment und der klinischen Erfahrung. Der Tierversuch hat den Wirkungsmechanismus dieser Mittel in vielen Punkten geklärt, für eine Reihe von ihnen - es sei nur an das Adrenalin erinnert - hat er erst die Grundlagen geschaffen, die eine rationelle Anwendung der betreffenden Substanzen am Krankenbett ermöglichen. Aber die Resultate derartiger Versuche am normalen oder krankgemachten Tier können naturgemäß nicht einfach auf den Menschen übertragen werden. Diese Feststellung kehrt in so vielen Arbeiten wieder, daß sie fast zum Gemeinplatz geworden-ist; merkwürdigerweise hat sie aber nur relativ selten zu der Folgerung geführt, auch beim Menschen außer der klinischen Beobachtung Methoden anzuwenden, die wie im Tierexperiment gestatten, die Wirkung des betreffenden Mittels in ihre einzelnen Komponenten aufzulösen. Wie wichtig es ist, diese Lücken auszufüllen, macht das Beispiel des schon erwähnten Adrenalins besonders deutlich: Beim Tier erfolgt nach der subcutanen Injektion dieser Substanz niemals eine Blutdrucksteigerung, beim Menschen scheint in vielen Fällen eine solche einzutreten.

Der kontinuierlichen Registrierung des art eriellen Blutdrucks, dieses wichtigsten Gradmessers für die Wirksamkeit eines Gefäßmittels im Tierversuch, stellen sich allerdings beim Menschen erhebliche Schwierigkeiten entgegen. Die direkte Druckmessung in einer Arterie kann nur in den seltensten Fällen angewandt werden, die indirekten Methoden sind, abgesehen davon, daß sie nur eine sehr unzulängliche Messung im a kuten Versuch erlauben, nicht imstande, das komplexe Ineinandergreifen der verschiedenen Gefäßprovinzen und des Herzens zu entwirren. 
Sehr viel günstiger liegen die Verhältnisse für die Bestimmung des venösen Blutdrucks. Beim Tier hat man diesem sicherlich nicht unwesentlichen Kreislauffaktor wenig Beachtung geschenkt: Fast alle Untersuchungen über Vasomotorenmittel lassen ihn und die Venen überhaupt ganz unberücksichtigt. Wieder kann das Beispiel des Adrenalins, einer experimentell-pharmakologisch doch gewiß gründlich studierten Substanz angeführt werden. In seiner umfassenden Darstellung bemerkt Biedl, daß die konstringierende Wirkung des Adrenalins auf die Venen, die übrigens keine erhebliche zu sein scheine, bisher nicht näher untersucht worden ist!

Beim Menschen ist die direkte Bestimmung des Venen. drucks durch graphische Registrierung der Druckschwankungen in einer Hautvene ${ }^{1}$ ) oder noch einfacher mit dem von Moritz ${ }^{2}$ ) angegebenen Verfahren möglich. Mit beiden Methoden kann man rasch eintretende Wirkungen von Vasomotorenmitteln verfolgen und messen.

Gelegentlich früherer Beobachtungen über die Wirkung des Adrenalins auf die Blutverteilung beim Menschen habe ich auf die erstgenannte Art das Verhalten des Venendrucks in seinen Beziehungen zur Volumkurve des Armes untersucht.

\section{Adrenalin.}

Nach intramuskulärer Injektion von Adrenalin tritt bei herzund gefäßgesunden Menschen, wie gezeigt werden konnte ${ }^{1}$ ), eine kurzdauernde Zunahme des Armvolumens ein, die sich plethysmographisch nachweisen läßt. Diese Volumzunahme ist nicht durch eine aktive Erweiterung der Armgefäße bedingt; sie kommt dadurch zustande, daß die an Masse und Kraft stärkeren und für Adrenalin empfindlicheren Gefäße des Splanchnicusgebietes einen Teil der in ihnen enthaltenen Blutmenge nach der Peripherie verschieben, wodurch die Extremitätengefäße passiv gedehnt werden.

Durch das Adrenalin werden nicht nur die Arterien, sondern auch die sympathisch innervierten Venen, die reichlich glatte Muskulatur besitzen, beeinflußt: gleichzeitige Registrierung der plethysmographischen Kurve und des Drucks in einer oberflächlichen Armvene ergab, daß der Ven en druck nach Adrenalininjektion ebenso wie das Armvolumen zunimmt. Beide Kurven verlaufen annähernd parallel.

Mit der angewandten Technik ließ sich die Änderung des Venendrucks nicht zahlenmäßig feststellen. Um hierüber genauen Aufschluß zu erhalten, habe ich deshalb Venendruckmessungen beim Menschen mittels des von Moritz angegebenen Verfahrens vorgenommen, das exakte Werte liefert.

1) Rosenow, Deutsches Archiv f. klin. Med. 12\%, 136. 1918.

2) Moritz u. v. Tabora, Deutsches Archiv f. klin. Med. 98, 475. 1910. 
Methodik: Zur Druckmessung wurde die Vena mediana des rechten Armes benutzt. Es wurde immer erst durch mehrmalige Messung der Venendruck festgestellt und dann die mit der Suprareninlösung (1: 1000) gefüllte Spritze in die Muskulatur des linken Oberarms eingestochen; nachdem die auf diesen Schmerzreiz meist erfolgende geringe Drucksteigerung abgeklungen war, wurde die Spritze langsam entleert. Injiziert wurde 0,4-1 mg Suprarenin. Nach der Injektion wurde in Abständen von $1 / 2-1$ Minute der Venendruck in der von Moritz angegebenen Weise gemessen. Die Dauer eines Versuches betrug 10 bis 36 Minuten.

Das aus den nachstehenden Tabellen und Kurven ersichtliche Ergebnis dieser Untersuchungen bestätigte zunächst, daß der normale

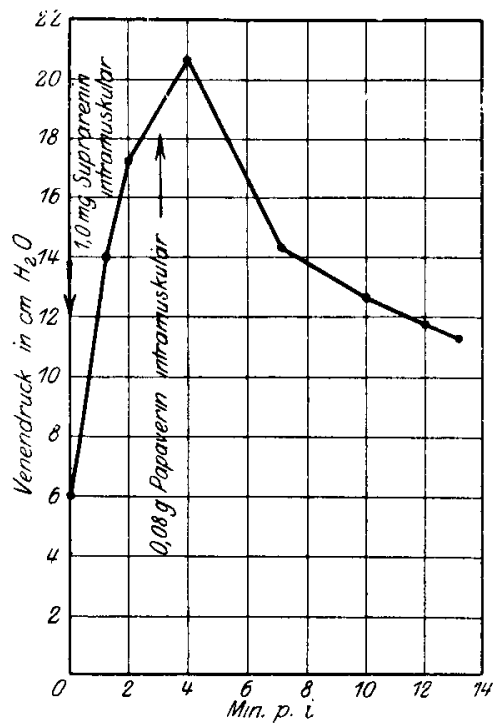

Abb. 1.

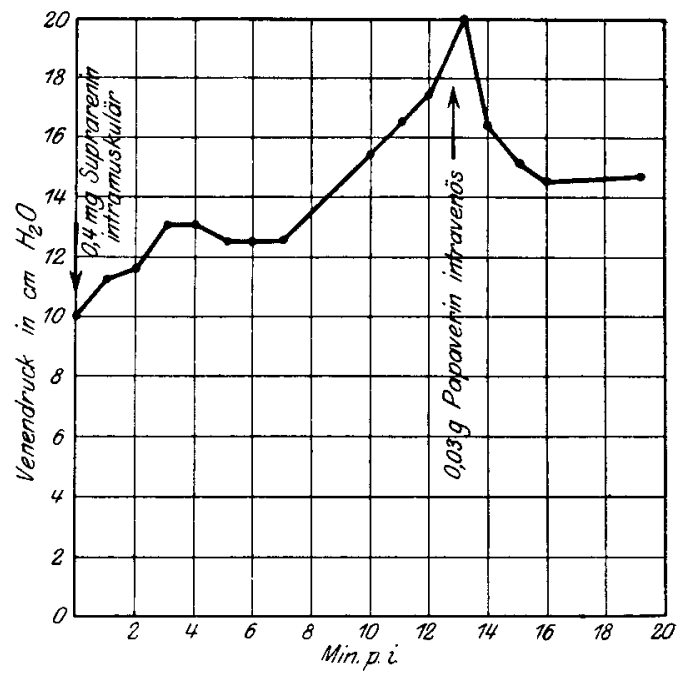

Abb: 2 .

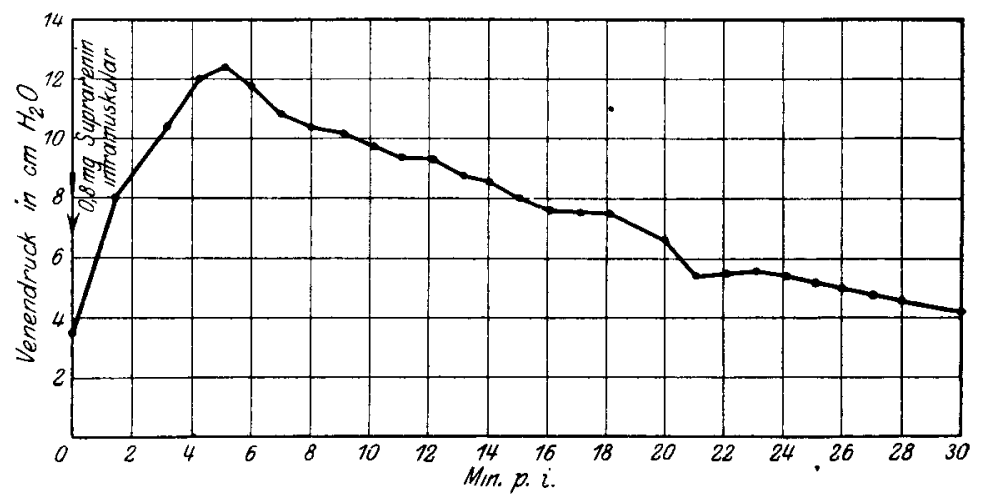

Abb. 3. 
Venendruck nach Adrenalininjektion ansteigt. Entsprechend der bekannten ${ }^{1}$ ) individuell verschiedenen Empfindlichkeit des Menschen für Adrenalin waren die Ausschläge verschieden groß, einige Male aber sehr beträchtlich. Die Drucksteigerung setzte gewöhnlich sehr rasch (etwa $1 / 2-1$ Minute) nach der Injektion ein und erreichte meist in 3-4 Minuten (zuweilen auch später) ihr Maximum. Die höchste beobachtete Steigerung von 6,2 auf $20,6(!) \mathrm{cm}$ Wasser erfolgte innerhalb 4 Minuten.

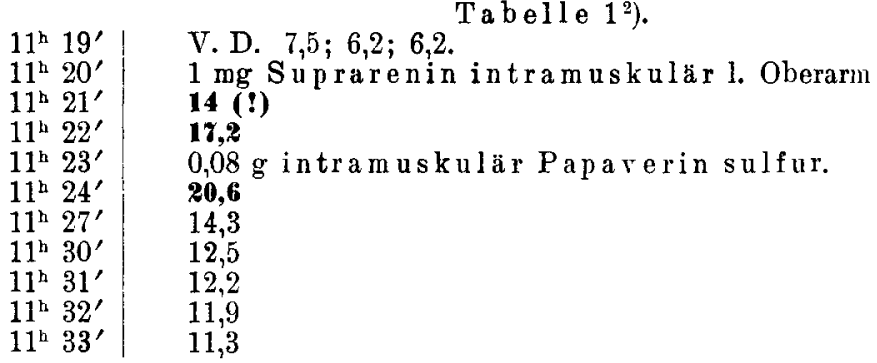

32 jahhr. Mann. Verdacht auf Ulcus ventriculi.

Sehr starke Reaktion. Fast unmittelbar nach der Injektion starke Kopfschmerzen. Blässe. Extrasystolen. Vaguspulse. Anscheinend erhebliche arterielle Drucksteigerung. Nach Papaverin $(0,08 \mathrm{~g}$ intramuskulär) rasch Abnahme der Beschwerden (siehe auch Kurve 1).

Die Wirkung des Adrenalins auf den Venendruck gleicht der auf das Armvolumen insofern, als auch das ven öse Druckmaxim u m meist nur kurze Zeit bestehen bleibt: sie unterscheidet sich nach den bisher untersuchten Fällen dadurch, daß das ursprüngliche Niveau des Venendrucks viel langsamer erreicht wird als das normale Armvolumen. Einige Male war innerhalb der Versuchsdauer der Venendruck nicht wieder auf seinen Anfangswert gesunken.

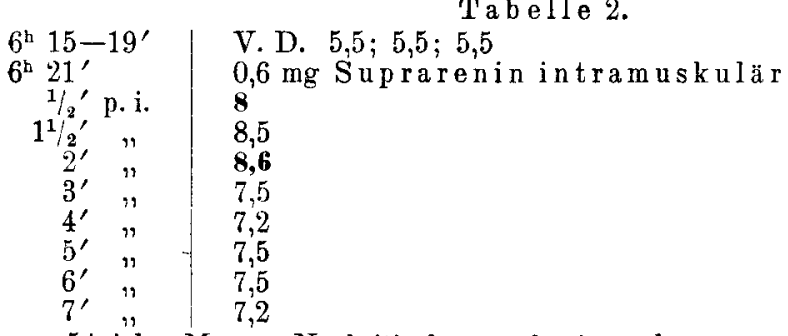

54 jähr. Mann. Nephritis haemorrhagica subacuta.

Keine subjektiven Beschwerden. Versuch wegen Zitterns des Kranken abgebrochen. Geringe Steigerung des Venendrucks.

1) Ba uer, Deutsches Archiv f. klin. Med. 10\%, 66. 1912; Fre y, Deutsche med. Wochenschr. 1917, S. 872; Dresel, Deutsche med. Wochenschr. 1919, S. 955.

2) Der Venendruck (V.D.) ist überall in $\mathrm{cm}$ Wasser, der arterielle Druc! in $\mathrm{mm}$ Quecksilber angegeben 
Tabelle 3 (siche Kurve 3).

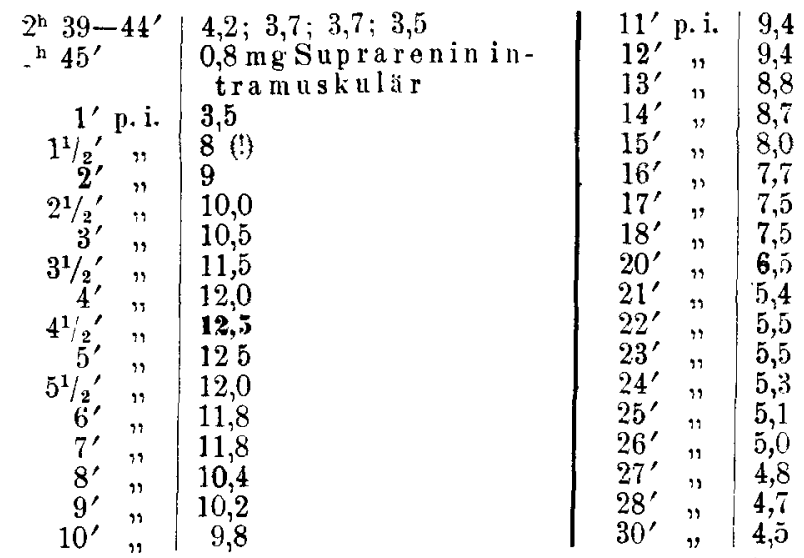

57 jähr. Mann. Emphysem. Chron. Bronchitis. Starke Adrenalinreaktion. tllmählicher Abfall des Venendrucks zur Norm in 30 Minuten.

\section{Tabelle 4 .}

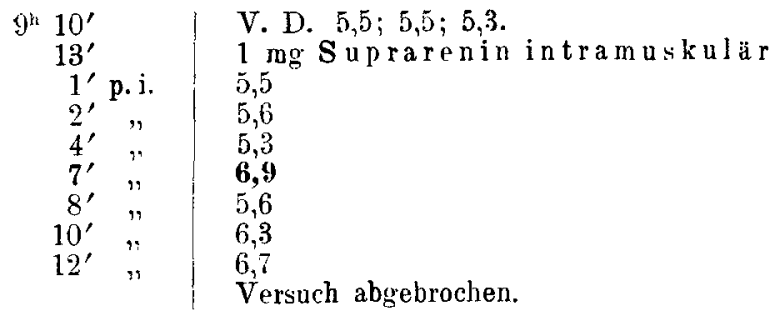

30 jăhr. Mann. Ohne krankhaften Befund. Geringe Adrenalinreaktion.

Wie die Steigerung des Venendrucks beim Menschen ${ }^{1}$ ) nach Adrenalininjektion zu erklären ist, läßt sich nicht ohne weiteres entscheiden. Die verschiedenen Möglichkeiten (vgl, auch 'Moritz l. c.) sollen in einer späteren Mitteilung erörtert werden. Die erhaltenen Resultate stützen jedenfalls die früher bereits ausgesprochene Auffassung, daß die plethysmographische Kurve auch bei kurzdauernden Schwankungen nur bedingt als Ausdruck arterieller Kaliberänderungen anzusehen ist. Sie sprechen dafür, daß namentlich, wenn sich der Druckim ge samten Gefäßsystem rasch und beträchtlich ändert, das venöse Stromgebiet auf die Gestaltung der Volumkurve von deutlichem Einflußsein kann.

1) Nicht verwertbar für die Beurteilung der akuten Gefäßwirkung des Adrenalins sind Beobachtungen von Ka u ert (D. Arch. f. klin. Med. 100, 398. 1910), der bei 4 Menschen den Venendruck unter dem Einfluß des Suprarenins langsam absinken sah. Das Minimum wurde nach 5-20 Minuten erreicht. Da aber der Druck nur in Intervallen von $10 \mathrm{zu} 10$ Minuten gemessen wurde, könnten etwaige flüchtige Druckänderungen gar nicht erkennbar werden. 


\section{Papaverin.}

Im Gegensatz zum Adrenalin, das auf die Gefäßmuskulatur exquisit tonussteigernd wirkt, setzt Papaverin den Tonus der glatten Muskulatur herab $\left.\left[\mathrm{Pa}^{1}\right)\right]$. Toxische Nebenwirkungen des Adrenalins können durch Papaverin schnell beseitigt werden (Rosenow l.c.). Es war deshalb anzunehmen, daß sich auch auf den durch Adrenalin erhöhten

\section{Tabelle 5 .}

\begin{tabular}{|c|c|c|c|}
\hline $10^{\text {h }} 17^{\prime}$ & $\begin{array}{r}\text { D. D. } 5,2 ; 5,3 ; 5,0 ; 5,2 ; 4,8 . \\
\text { Art. Blutdruck } 90-95 \mathrm{~mm}\end{array}$ & & $\begin{array}{l}0,04 \mathrm{~g} \text { Papaverin intra } \\
\text { venös }\end{array}$ \\
\hline & Hg. & $13^{\prime}$ p.i. & 9,5 \\
\hline $10^{\text {h }} 25^{\prime}$ & V. D. 5,2 & $14^{\prime} "$ & 9,0 \\
\hline $10^{\mathrm{h}} 25^{\prime}$ & $\begin{array}{l}0,6 \mathrm{mg} \text { Suprarenin in - } \\
\text { tramuskulär }\end{array}$ & $\begin{array}{l}15^{\prime}, " \\
16^{\prime}, "\end{array}$ & $\begin{array}{l}9,4 \\
9,7\end{array}$ \\
\hline $1^{\prime}$ p. i. & 5,2 & $17^{\prime \prime \prime \prime}$ & 9,5 \\
\hline $2^{\prime}$ & 6,4 & $18^{\prime}$ & 9,2 \\
\hline $3^{\prime}$ & 7,4 & $19^{\prime}$ & 9,2 \\
\hline $4^{\prime}$ & 8,0 & $20^{\prime}$ & 8,3 \\
\hline $5^{\prime}$ & 8,6 & $21^{\prime}, "$ & 8,3 \\
\hline $6^{\prime}$ & 8,3 & $22^{\prime}$ & 8,3 \\
\hline $7^{\prime}$ & 8,3 & $23^{\prime}$ & 7,7 \\
\hline $8^{\prime}$ & 8,5 & $24^{\prime}$, & $8, \overline{5}$ \\
\hline $9^{\prime}$ & 9,3 & $25^{\prime} n$ & Art. Druck 105-110 \\
\hline $10^{\prime}, "$ & 9,4 & & \\
\hline $11^{\prime}, n$ & 9,4 & & \\
\hline $12^{\prime} \quad$, & 9,2 & & \\
\hline
\end{tabular}

27 jähr. Mann. Angina pectoris vasomotoria nach Nicotinabusús (40-50 Zigaretten täglich). Außerhalb des Anfalls. Geringe Adrenalinwirkung, Papaverin ohne deutlichen Einfluß. (Vgl. Tab. 6).

Tabelle 6 (siehe auch Kurve 2).

\begin{tabular}{|c|c|}
\hline $10^{\mathrm{h}} 46-48^{\prime}$ & $\begin{array}{l}\text { V. D. } 10,2 ; 10,6 ; 9,8 \text {. Art. } \\
\text { Blutdruck } 100-105\end{array}$ \\
\hline $10^{\mathrm{h}} 50^{\prime}$ & $\begin{array}{l}0,4 \text { mg Suprarenin } \\
\text { intramuskulär }\end{array}$ \\
\hline $1^{\prime}$ p.i. & 11,2 \\
\hline $2^{\prime} \quad$, & 11,5 \\
\hline & 13,0 \\
\hline & 13,0 \\
\hline $5^{\prime} \quad$, & 12,5 \\
\hline $6^{\prime}$ & 12,5 \\
\hline $7^{\prime}$ & 12,5 \\
\hline $10^{\prime}$ & 15,5 \\
\hline
\end{tabular}

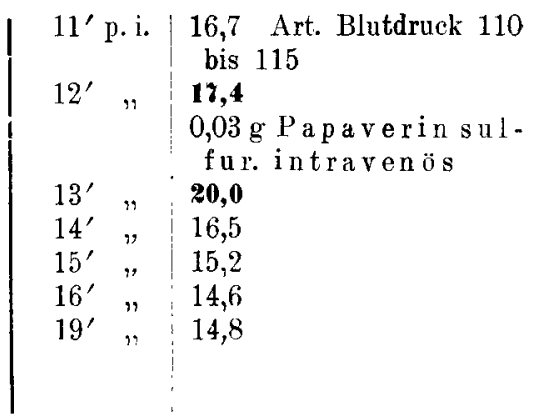

27 jähr. Mann. Angina pectoris vasomotoria. Die Druckmessung wurde während eines Anfalls vorgenommen. Suprarenin beseitigte die vorher sehr heftigen Schmerzen sofort! Papaverin ohne wesentlichen Einfluß. (Vgl Tab. 5). S. 1796 .

1) Pal, Wiener med. Wochenschr. 1913, S. 1050 u. 2513;' Med. Klin. 1913, 
Venendruck ein entsprechender Einfluß des Papaverins geltend machen würde. In der Tat sanken die hohen Druckwerte nach intravenöser Papaverininjektion zuweilen auffallend rasch ab; gleichzeitig schwanden die etwa nach Adrenalin aufgetretenen Beschwerden (vgl. u. a. Abb. 2 und Tab. 1).

$$
\text { T'abelle } 7 \text {. }
$$

\begin{tabular}{|c|c|c|c|c|}
\hline $2^{\mathrm{h}} 52-55^{\prime}$ & V. D. 9,$8 ; 9,3 ; 9,3$. Art. & $13^{\prime} \mathrm{p}$ & p. i. & 11,7 \\
\hline & Druck $115-120$ & $14^{\prime}$ & $"$ & 11,5 \\
\hline $2^{h_{4}} 55^{\prime}$ & $0,5 \mathrm{mg}$ Suprarenin & $15^{\prime}$ & $"$ & 11,2 \\
\hline & intramuskulär & $16^{\prime}$ & $n$ & 11,3 \\
\hline $1^{\prime}$ p.i. & 9,3 & $17^{\prime}$ & 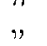 & 11,2 \\
\hline $2^{\prime} n$ & 9,0 & $3^{\text {in }} 15^{\prime}$ & & 0,04 g Papaverin in \\
\hline $3^{\prime}$ & 8,5 & & & trarenös \\
\hline $4^{\prime}$ & 8,8 & $1^{\prime}$ & $"$ & 13,5 \\
\hline $5^{\prime}$ & \begin{tabular}{|l}
8,2 \\
\end{tabular} & $2^{\prime}$ & $n$ & 12,6 \\
\hline & $\begin{array}{l}\text { 0,4 mg Suprarenin in- } \\
\text { tramuskulär }\end{array}$ & $\begin{array}{l}4^{\prime} \\
5^{\prime}\end{array}$ & $"$ & $\begin{array}{l}12,6 \\
11,6\end{array}$ \\
\hline $6^{\prime}$ & 10 & $6^{\prime}$ & $"$ & 11,4 \\
\hline $7^{\prime}$ & 11,3 & $7^{\prime}$ & $n$ & 10,6 \\
\hline $8^{\prime}$ & 12,3 & $8^{\prime}$ & $n$ & 10,6 \\
\hline $9^{\prime}$ & 12,7 & $9^{\prime}$ & יוּי & 10,8 \\
\hline $10^{\prime}$ & 13,1 & $10^{\prime}$ & $n$ & 9,7 \\
\hline $11^{\prime}$ & 12,0 & $11^{\prime}$ & $n$ & 9,7 \\
\hline $12^{\prime}$ & 11,6 & $12^{\prime}$ & $"$ & 9,5 \\
\hline
\end{tabular}

33 jähr. Mann. Alte Hilustuberkulose. Neurastheniker.

$0,4 \mathrm{mg}$ Suprarenin sind wirkungslos bzw. machen geringe Drucksenkung! Nochmalige Injektion der gleichen Dosis steigert den Venendruck bis 13,1 . Papaverin anscheinend ohne wesentlichen Einfluß. Anfangs Steigerung nach Papaverin (Schmerzreaktion!).

In anderen Fällen war eine solche Wirkung nicht erkennbar, der Venendruck blieb auch nach der Papaverininjektion hoch oder stieg sogar noch kurze Zeit weiter an (s. die vorstehenden Tab. 5, 6, 7).

Der Venendruck des Gesunden wird durch Papaverin wenig verändert; dagegen ließ sich eine mäßige Senkung des Venendrucks wiederholt bei arterieller $\mathrm{Hy}$ pertonie erzielen; besonders deutlich erkennbar war diese Wirkung bei intravenöser Injektion des Mittels.

$$
\text { Tabelle } 8 \text {. }
$$

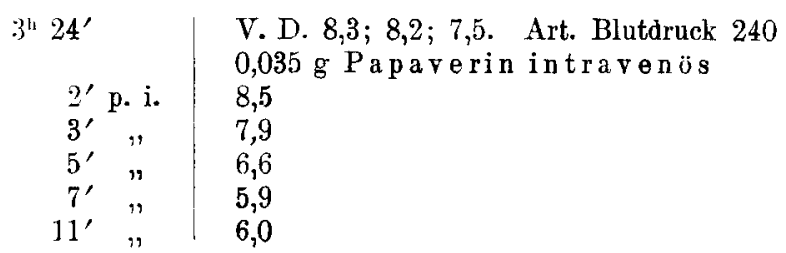

Art. Blutdrack unverăndert. 
Tabelle 8 a.

\begin{tabular}{|c|c|c|c|}
\hline $2 \mathrm{Tage}$ & & & \\
\hline $10^{1} 58^{\prime}$ & V. D. 4,$5 ; 4,1 ; 4, \overline{7}$ & $8^{\prime}$ p.i & 2,7 \\
\hline $11^{\mathrm{h}} 5^{\prime}$ & $\begin{array}{l}0,024 \mathrm{~g} \text { Papaverin intra- } \\
\text { venös }\end{array}$ & $\begin{array}{l}12^{\prime} ", \\
14^{\prime} " \prime\end{array}$ & $\begin{array}{l}2,1 \\
2,5\end{array}$ \\
\hline $1 / 2^{\prime}$ p. i. & 3,8 & $16^{\prime} "$ & $4,9(!)$ \\
\hline $1^{\prime}$ & 4,2 & $17^{\prime}, "$ & $5,5(!)$ \\
\hline $2^{\prime} n$ & 4,2 & $19^{\prime}$, & 5,1 \\
\hline $3^{\prime}$ & 3,4 & $20^{\prime}, n$ & 4,8 \\
\hline $4^{\prime} n$ & 3,7 & $22^{\prime} n$ & 4,7 \\
\hline $5^{\prime}$ & 3,3 & $24^{\prime} "$ & 3,8 \\
\hline $6^{\prime}$, & 3,2 & $25^{\prime} " \prime$ & 3,8 \\
\hline $7^{\prime} "$ & 2,7 & $27^{\prime} " \prime$ & 3,3 Art. Blutdruck $190-195$ \\
\hline
\end{tabular}

56 jähr. Mann. Nephritis chronica. Hypertonie. Urämische Dyspnöe. Nach Papaverin mehrere Tage anhaltende subjektive und objektive Besserung (siehe Kurve 4).

Tabelle 9 .

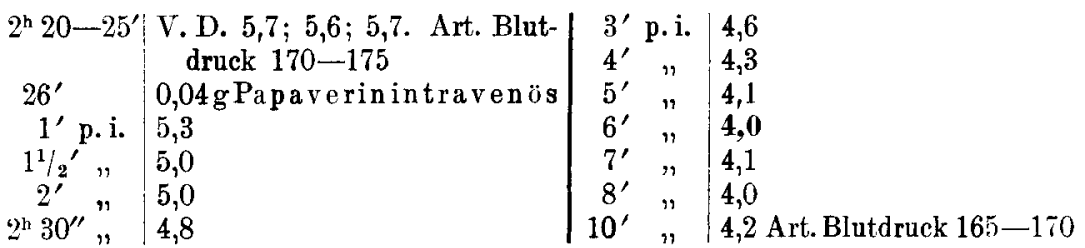

54 jähr. Mann. Nephritis chronica.

Geringe Papaverinwirkung.

Die intramuskuläre Injektion wirkt im gleichen Sinne, aber weniger kräftig.

Tabelle 10 .

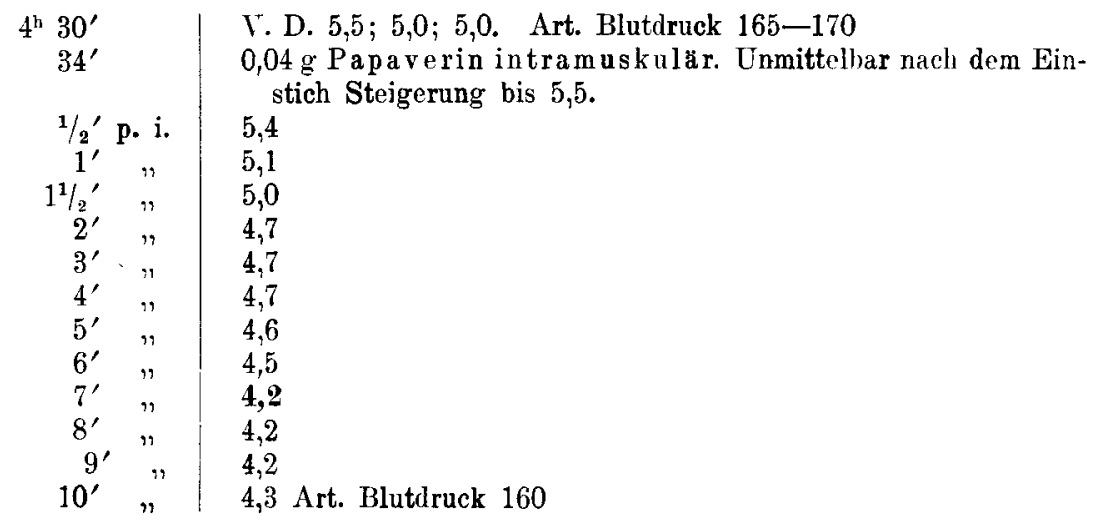

50 jähr. Mann. Nephritis acuta haemorrhag. Keine Erhöhung des Venendrucks. Geringe Papaverinwirkung. 
Tabelle 11.

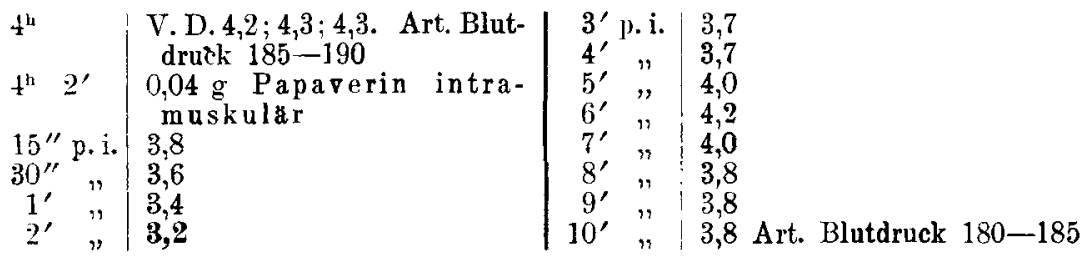

28jähr. Mann. Nephritis subacuta haemorrhag. Normaler Venendruck. Geringe Papaverinwirkung.

Besonders bemerkenswert ist Abb. 4 (vgl. Tab. 8a). Die intravenöse Papaverininjektion führte bei dem Kranken, der an nephritischer Hypertonie litt, zunächst zu einer geringen Abnahme des Venendrucks; 15 Minuten p. i. folgte plötzlich eine erhebliche Steigerung über den Ausgangswert, die erst im Verlauf von weiteren 13 Minuten allmählich schwand. Man wird wohl in diesem Fall abnorm starke Tonusschwankungen der Gefäße annehmen müssen. Dafür spricht auch das sehr wechselnde Verhalten des arteriellen Drucks bei diesem Kranken (240-190 $\mathrm{mm} \mathrm{Hg}$ an einem Tag!).

Einige Male wurde bei chronischen Nephritiden vor und nach Papaverininjektion der arterielle Blutdruck bestimmt; er schien $\mathrm{zu}$ w $\mathrm{i}$ le n nach der Injektion des Mittels etwas abzusinken. Der n or male arterielle Druck wird dagegen, wie schon $\mathrm{Pal}^{1}$ ) betont hat, nicht oder kaum beeinflußt. Das Papaverin beseitigt offenbar nur den Teil der nephritischen Hypertonie, der auf spastischen Zuständen der Gefäße beruht.

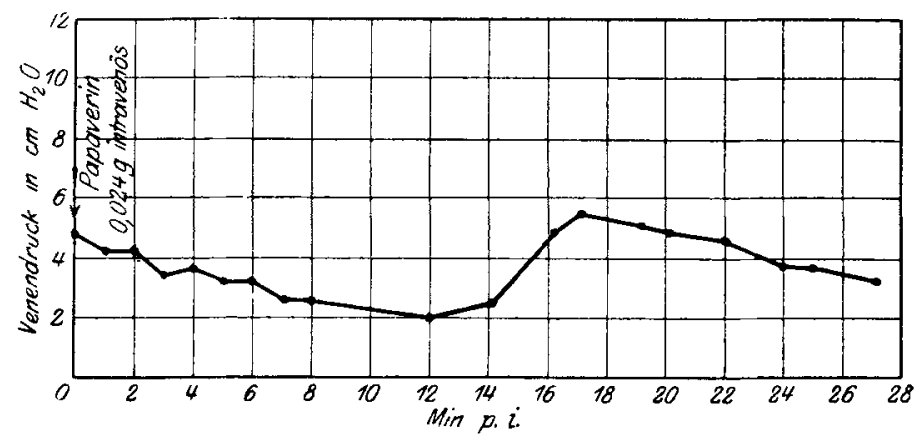

Abb. 4 .

Die flüchtige Wirkung des Papaverins (s. Kurven) läßt therapeutische Versuche bei Hypertonie nicht aussichtsreich (vgl. auch Pal l. c.) erscheinen, dagegen kann man die Substanz bei urämischer Dyspnöe, wie es scheint mit Nutzen verwenden. Ein Kranker (s. Abb. 4, Tab. 8a)

1) Pal, l.. 
mit hochgradigen Beschwerden gab spontan an, daß ihm die Papaverininjektion in ähnlicher Weise Erleichterung verschafft habe wie früher ein Aderlaß.

\section{Strychnin.}

Das Adrenalin greift vorwiegend peripher an; der Typus eines zentralen. Vasomotorenmittels ist das Strychnin. In letzter Zeit ist dieses in Amerika und Frankreich viel benutzte Alkaloid auch bei uns von verschiedenen Autoren warm zur Bekämpfung von Kreislaufstörungen - namentlich toxisch-infektiöser Vasomotoren. schwäche ${ }^{1}$ ) - empfohlen worden. Seine Anwendung erscheint auch durchaus rationell, namentlich in Kombination mit anderen Gefäßmitteln, die ihren Angriffspunkt in der Peripherie haben, z. B. Adrenalin $\left[\right.$ F r e $\left.\left.y^{2}\right)\right]$. Strychnin hat vor dem Adrenalin den Vorteil, daß es unbedenklich in Dosen von $2-3 \mathrm{mg}$ in traven ös injiziert werden kann. Eine etwaige Wirkung auf den Venendruck müßte bei dieser Art der Applikation besonders deutlich hervortreten.

Wie die nachstehenden Protokolle (Tab. $12 \mathrm{u} .13$ ) zeigen, änderte sich aber der Venendruck fast gar nicht, während Suprarenin (intramuskulär) den Venendruck erheblich steigerte. Daß der Venendruck durch Strychnin unbeeinflußt bleibt (die geringfügige Senkung kommt nicht in Betracht), spricht nicht gegen die oben erwähnte therapeutische Verwendung des Mittels. Es wird ja gerade für die Fälle empfohlen, bei denen eine pathologisch geänderte Blutverteilung besteht (Kollaps, Kreislaufsehwäche bei akuten Infektionen). Es wäre durchaus denkbar, daß das Strychnin bei solchen Kranken etwaige hohe Venendruckwerte erniedrigt. Ich habe zu einer Prüfung dieser Frage bisher keine Gelegenheit gehabt.

\begin{tabular}{|c|c|c|c|}
\hline \multicolumn{4}{|c|}{ Tabelle 12.} \\
\hline $\begin{array}{l}3^{\mathrm{h}} 24^{\prime} \\
26^{\prime}\end{array}$ & $\begin{array}{l}\text { Y. D. } 5,5-6 ; 5,5-6 ; 5,5-6 \\
5,5-6\end{array}$ & $3^{\text {h }} 32^{\prime}$ & $\begin{array}{l}1 \text { mg Suprarenin intra- } \\
\text { muskulär }\end{array}$ \\
\hline $3^{\text {h }} 26^{\prime}$ & $\begin{array}{l}2 \mathrm{mg} \text { Strychnin nitric. } \\
\text { intravenos }\end{array}$ & ${ }^{1 / 2}{ }^{\prime} p . i$. & $\begin{array}{l}5,5-6 \\
5,5-6\end{array}$ \\
\hline $1 / 2^{\prime}$ p.i. & $4,5-5$ & $11 / 2^{\prime}$ & $6-6,5$ \\
\hline $1^{\prime}$ & $4,5-5$ & $2^{\prime}$ & $8-9$ \\
\hline $1 / 2^{\prime}$ & $5,5-6$ & $2^{1 / 2^{\prime}}$, & $7,5-8$ \\
\hline $2^{\prime}$ & $5-5,5$ & $3^{\prime}$ & 7,5 \\
\hline $3^{\prime}$, & 5,5 & $3^{1} / 2^{\prime}$ & 7 \\
\hline $4^{\prime}$ & 5,0 & $4^{\prime}$ & $6,5-7$ \\
\hline $5^{\prime}$ & 5,5 & $41 / 2^{\prime} \quad$, & $6-6,5$ \\
\hline & & $\begin{array}{l}5^{\prime}, ", \\
511,\end{array}$ & \\
\hline & & $\begin{aligned} 51 / 2 & = \\
6^{\prime} & " 1\end{aligned}$ & $\begin{array}{l}6-6,5 \\
5,5-6\end{array}$ \\
\hline
\end{tabular}

32 jähr. Mann. Pneumonie des r. Unterlappens.

1) O. Schiffner, Med. Klinik 1919, S. 973 (dort Lit.!).

2) W. Frey, Berl. klin. Wochenschr. 1919, S. 148. 
Über die Wirkung von Gefảßmitteln auf den Venendruck des Menschen. 343

$$
\text { Tabell e } 13 .
$$

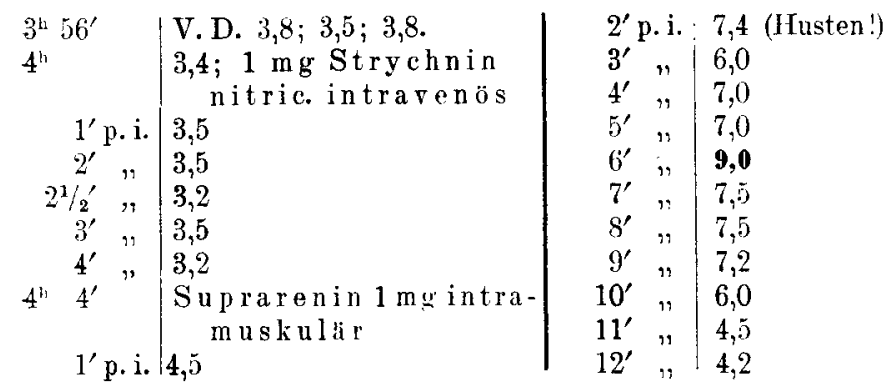

48 jähr. Mann. Abgelaufene Pneumonie des Mittellappens. 
(Aus der Medizinischen Klinik der Universität Königsberg.)

\section{Uber die Wirkung von Gefäßmitteln auf den Venendruck des Mensehen.}

2. Mitteilung. Hypophysenextrakte.

Von

Privatdozent Dr. Georg Rosenow.

(Eingegangen am 7. Januar 1920.)

Während die hämodynamischen Wirkungen der Nebennieren extrakte durch den Tierversuch nahezu restlos geklärt sind, hat die entsprechende experimentelle Analyse der Hypophysenextrakte bisher noch nicht zu eindeutigen Ergebnissen geführt. Die Gründe sind klar : Dort kann man mit dem isolierten wirksamen Prinzip der Nebenniere, dem chemisch gut definierten Adrenalin arbeiten, hier, bei der Hypophyse, ist man auf Extrakte angewiesen, die Gemische von Körpern ganz verschiedener physiologischer Wertigkeit sind. Auch das Hy pophysin des Handels, bei dem zuerst erfolgreiche Versuche angestellt wurden, die wirksamen Bestandteile aus der Drüse krystallisiert zu erhalten ${ }^{1}$ ), ist keine einheitliche Substanz, sondern ein Gemisch.

Alle im Handel befindlichen Hypophysenpräparate, wie das Pituglandol, Pituitrin, Hypophysin, Coluitrin, entstammen dem Hinterlappen oder der Pars intermedia der Drüse; sie steigern im (Gegensatz zu den Vorderlappenextrakten ${ }^{2}$ ) a m Tier den arteriellen Blutdruck durch Vasoconstriction und Verbesserung der Herzarbeit ${ }^{3}$ ). Insofern ist also eine Analogie zu der markantesten Wirkung des Adrenalins vorhanden. Aber'diese Ähnlichkeit ist nur oberflächlich; es bestehen tatsächlich erhebliche qualitative und quantitative Unterschiede: Die Blutdrucksteigerung nach Injektion der Hypophysenextrakte fällt schwächer aus als nach der des Adrenalins, sie klingt aber viel langsamer ab. Beim Adrenalin ruft jede neue Injektion von neuem einen Druckanstieg hervor, bei den Hypophysenextrak-

1) Füh ner, Münch. med. Wochenschr. 1912, S. 568; Deutsche med. Wochenschr. 1913, S. 491; Zeitschr. f. d. ges. experim. Med. 1, 397. 1913.

$\left.{ }^{2}\right)$ Ha mburger, Amer. Journ. of Physiol. 26, 178. 1911. - Biedl, Innere Sekretion. 3. Aufl. 1916. II, S. 141.

$\left.{ }^{3}\right)$ Oliver und Schaefer, Journ. of Physiol. 1895, S. 277. 
ten wirken die der ersten Injektion, in kurzem Abstand folgenden, nicht drucksteigernd ${ }^{1}$ ), sondern blutdrucksenkend ${ }^{2}$ ).

Die Reizwirkung auf den Accelerans fehlt den Hypophysenextrakten vollständig ${ }^{3}$ ), sie rufen vielmehr Bradykardie ${ }^{4}$ ) hervor. Weitere Unterschiede sind onkometrisch [Magn us und Schaefer $\left.\left.{ }^{5}\right)\right]$, an isolierten Organen [Wiggers $\left.\left.{ }^{6}\right)\right]$ und Gefäßen $\left.\left[\mathrm{Pal}, \mathrm{Cow}^{7}\right)\right]$ aufgedeckt worden. Splanchnicus- und Coronargefäße scheinen entgegengesetzt beeinflußt zu werden wie durch Adrenalin: Die Gefäße des Splanchnicusgebiets, die Adrenalin verengert, werden z. T. durch Hypophysenextrakt dilatiert, die Coronargefäße, die Adrenalin erweitert, kontrahiert.

Freilich sind alle diese Effekte nicht konstant zu erzielen. Als sicher darf jedenfalls angenommen werden, daß in den Extrakten des Handels vasoconstrictorische und vasodilatorische Substanzen enthditen sind, von denen bei Erstinjektion im Tierversuch gew öhnlich diegefäßverengernden überwiegen.

Uber die kardiovasculäre Wirkung der Hypophysenextrakte beim Menschen ist bisher sehr wenig bekannt. Zu der Schwierigkeit, die in der Anwendung eines so komplexen Gemischs liegt, kommen hier die vorläufig noch unübersehbaren Empfindlichkeitsunterschiede. Die therapeutische Verwertbarkeit der Hypophysenpräparate als Gefäßmittel ist deshalb auch nicht unbestritten. Der warmen Empfehlung von $\mathrm{Klotz}$ und $\mathrm{Jaschk}^{8}$ ), die Hypophysenextrakt als Analepticum bei toxisch-infektiöser Blutdrucksenkung wirksam fanden, stehen Erfahrungen von Behrenroth ${ }^{9}$ ) und v.d. Velde $n^{10}$ ) gegenüber, wonach von einer irgendwie konstanten Wirkung auf den Blutdruck (allerdings beim nor malen Menschen) nicht gesprochen werden kann. In jüngster Zeit hat Lesch ke ${ }^{11}$ ) die Beeinflussung des Kreislaufs durch Hypophysenhinterlappenextrakt (Hypophysin) beim gesunden Menschen und bei Patienten mit nervöser Tachykardie untersucht. Er fand nach intravenöser Injektion größerer Mengen $(5-10 \mathrm{ccm}) \mathrm{Hypophysin} \mathrm{in}$ den meisten (wenn auch keineswegs in allen) Fällen Bradykardie und Blutdrucksenkung von verschieden langer Dauer. Es herrscht also

1) Howell, Journ. of experim. Med. 3, 215 u. 245. 1898.

2) Schaefer und Vincent, Journ. of Physiol. 25, 87. 1899.

3) Oliver und Schaefer, 1. c.

4) Howell, 1. c.

5) Magnus und Schaefer, Journ. of Physiol. 27, 44. 1901/02.

6) Wiggers, Amer. Journ. of med. Science 1911, S. 502.

7) Pal, Wiener med. Wochenschr. 1909, S. 137.-Cow, Journ. of Physiol. 42, 125. 1911.

8) Klotz, Med. Klin. 1913, S. 992; Monatssohr. f. Gynäkol. 36, Ergänzungsheft S. 91. 1912; J a s chke, Münch. med. Wochenschr. 1912, S. 1663.

9) Deutsches Archiv f. klin. Med. 113, 393. 1914.

10) Berl. klin. Wochenschr. 1913, S. 2084.

11) Zeitschr. f. klin. Med. 88, 225. 1919. 
keineswegs Ubereinstimmung, welche hämodynamischen Wirkungen beim Menschen die Injektion dieser Mittel im Gefolge hat.

Einen Einblick in den Mechanismus der Kreislaufswirkung konnte man von der Bestimmung des Venendrucks erwarten, zumal im Vergleich mit seiner Beeinflussung durch Adrenalin. Ich habe deshalb derartige Untersuchungen beim Menschen mit der beschriebenen Versuchsanordnung (s. die vorstehende Mitteilung) vorgenommen. Verwandt wurde fast ausschließlich Pituglandol ${ }^{1}$ ) (Chemische Werke Greuzach); das Präparat wurde teils intramuskulär, teils intravenös injiziert.

Wie die nachstehenden Protokolle zeigen, war die Wirkung a uf den Venendruck eine wesentlich andere als nach Injektion des Adrenalins. In den meisten Fällen sank der Venendruck während der Messung deutlich ab. Die Senkung erfolgte stets allmählich, niemals plötzlich, auchnicht bei intrarenöser Injektion. Das Zeitintervall, in dem das Druckminimum erreicht wurde, war verschieden groß, ebenso die relative Größe der Druckabnahme.

\begin{tabular}{|c|c|c|c|}
\hline \multicolumn{4}{|c|}{ Tabelle 1.} \\
\hline \multicolumn{2}{|c|}{ 29. XI. } & $12^{\prime}$ & 2,4 \\
\hline $5^{\text {h }} 12^{\prime}$ & Art. Blutdruck 120. & $13^{\prime}$ & 2,4 \\
\hline $5^{\mathrm{h}} 15^{\prime}$ & V. D. 4,$7 ; 4,8 ; 4,8$ & $14^{\prime}$ & 3,2 \\
\hline $5^{\text {ln }} 20^{\prime}$ & $\left.1 \mathrm{ccm}^{2}\right)$ Pituglandol in - & $15^{\prime}$ & 2,2 \\
\hline & tramuskulär. & $16^{\prime}$ & 2,0 \\
\hline p.i. $l^{\prime}$ & 4,3 & $17^{\prime}$ & 1,8 \\
\hline $2^{\prime}$ & 3,7 & $18^{\prime}$ & 1,5 \\
\hline $3^{\prime}$ & 2,9 & $19^{\prime}$ & 1,6 \\
\hline $4^{\prime}$ & 3,0 & $5^{\text {h }} 47^{\prime}$ & V. D. 2,$4 ; 0,5$ mg Supra. \\
\hline $5^{\prime}$ & 2,7 & & renin intramuskulär. \\
\hline $6^{\prime}$ & 2,6 & p. i. $\mathbf{4}^{\prime}$ & 5,2 \\
\hline $7^{\prime}$ & 2,8 & $5^{\prime}$ & 4,7 \\
\hline $8^{\prime}$ & 2,6 & $6^{\prime}$ & 4,3 \\
\hline $9^{\prime}$ & 3,0 & $7^{\prime}$ & 4,3 \\
\hline $10^{\prime}$ & 2,8 & $8^{\prime}$ & 4,2 \\
\hline $11^{\prime}$ & 2,7 & & Art. Blutdruck 120. \\
\hline
\end{tabular}

32jähr. Mann. Rekonvaleszenz nach Pneumonie.

Tabelle 2.

1. XII.

$\begin{array}{r:l}4^{\mathrm{h}} 55^{\prime} & \text { Art. Blutdruck } 130 . \\ 4^{\text {h }} 58^{\prime}-5^{\mathrm{h}} 2^{\prime} & \text { V. D. 5,5; 5,8; 5,8. } \\ 5^{\mathrm{h}} 5^{\prime}-5^{\mathrm{h}} 6^{\prime} & \text { 1 cem Pituglandol in- } \\ & \text { travenös. } \\ 5^{1} 7^{\prime} \text { p. i. } \mathbf{1}^{\prime} & 5,8 \\ 2^{\prime} & 5,8 \\ 3^{\prime} & 5,8\end{array}$

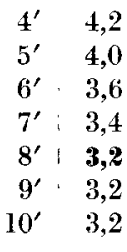

32jähr. Mann. Rekonvaleszenz nach Pneumonie (s. auch Tab. 1).

1) Hypophysin, das einige Male benutzt wurde, wirkt gleichsinnig.

2) $1 \mathrm{ccm}$ Pituglandol entsprechend 0,2 g Drüsensubstanz. 
I'ber die Wirkung von Gefámitteln auf den Venendruck dis Menschen. 347

Tabelle 3.

\begin{tabular}{r|l|r|r}
$5^{\text {h }} 10^{\prime}$ & art. Blutdruck 100. & $6^{\prime}$ & 4,3 \\
& V. D. 5,2; 4,5; 4,4. & $7^{\prime}$ & $\mathbf{4 , 0}$ \\
$5^{\text {h }} 15^{\prime}$ & I ccm Pituglandol intramus- & $8^{\prime}$ & 4,5 \\
& kulär. & $9^{\prime}$ & $\mathbf{4 , 3}$ \\
p. i. $1^{\prime}$ & 3,8 & $10^{\prime}$ & 4,3 \\
$2^{\prime}$ & $\mathbf{4 , 2}$ & $13^{\prime}$ & $\mathbf{4 , 0}$ \\
$3^{\prime}$ & 4,2 & $12^{\prime}$ & $\mathbf{3 , \gamma}$ \\
$4^{\prime}$ & 4,4 & $13^{\prime}$ & 3,7 \\
$5^{\prime}$ & $\mathbf{4 , 4}$ & $14^{\prime}$ & 3,8 \\
$15^{\prime}$ & 3,6 & &
\end{tabular}

48 jähr. Mann. Abgelaufene Pneumor.ie d. r. Unterlappens.

Besondere Besprechung erfordern die Fälle, in denen keine Druckabnahme nach dex Injektion eintrat.

Eine Steigerung des Venendrucks sah ich nur zweimal (Tab. 4 und 5). Der eine Kranke war ein ausgesprochener Neurastheniker, bei dem nach intramuskulärer Injektion von Pituglandol der Venendruck von $4,2-6,8 \mathrm{~cm}$ stieg; nach $0,5 \mathrm{mg}$ Suprarenin erfolgte

Tabclle 4.

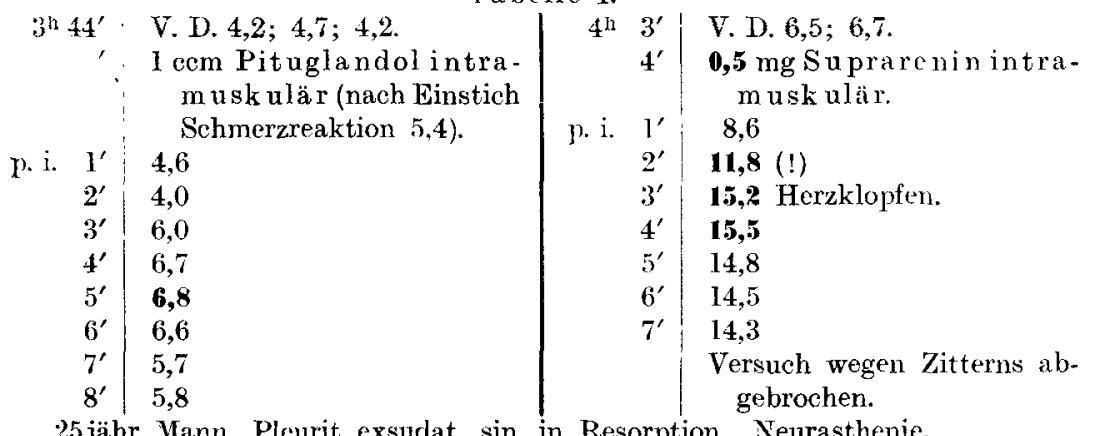

25jähr. Mann. Pleurit. exsudat. sin. in Resorption. Neurasthenie.

Tabelle 5 .

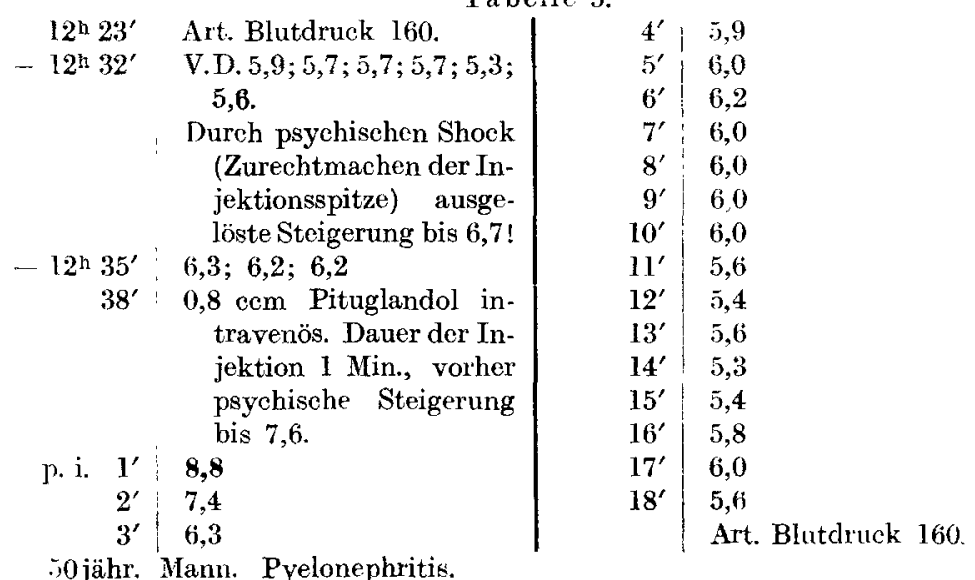


dagegen ein Anstieg von 6,5 auf 15,5 cm! Auch in dem anderen Fall (Tab. 5), bei dem der Venendruck eine kurzdauernde geringe Steigerung zeigte (von 6,3-8,8 cm), handelte es sich um einen vasomotorisch sehr labilen Menschen, wie die vor der Injektion psychisch ausgelösten Druckschwankungen zeigen.

Auffallend gering war der Einfluß des Pituglandols bei einem Urämischen mit primär hohem Venendruck (Tab. 6, 7, 8). Keine Tabelle 6 .

\begin{tabular}{|c|c|c|c|}
\hline \multicolumn{2}{|c|}{ 25. XI. } & $6^{\prime}$ & $13,0-13,4$ \\
\hline \multirow{3}{*}{$5^{\mathrm{h}} 45^{\prime}$} & Art. Blutdruck 170. & $7^{\prime}$ & $13,0-13,3$ \\
\hline & V. D. 14,$7 ; 14,2 ; 14,2 ; 13,5$ & $8^{\prime}$ & $12,8-13,0$ \\
\hline & (großeAtemschwankungen!). & $9^{\prime}$ & $12,8-13,0$ \\
\hline \multirow[t]{2}{*}{$5^{\text {h }} 47^{\prime}$} & $1 \mathrm{ccm}$ Pituglandol in- & $10^{\prime}$ & 13,0 \\
\hline & tramuskulär. & $11^{\prime}$ & 13,0 \\
\hline \multirow[t]{5}{*}{ p. i. } & $14,5-15,0$ & $12^{\prime}$ & 13,3 \\
\hline & $14,5-15,0$ & $13^{\prime}$ & 12,7 \\
\hline & $13,5-14,2$ & $14^{\prime}$ & 12,9 \\
\hline & $13,2-13,7$ & $15^{\prime}$ & 12,7 \\
\hline & $13,2-13,5$ & & Art. Blutdruck 170. \\
\hline
\end{tabular}

43 jähr. Mann. Akute Nephritis. Urämie (s. auch Tab. 7 u. 8.
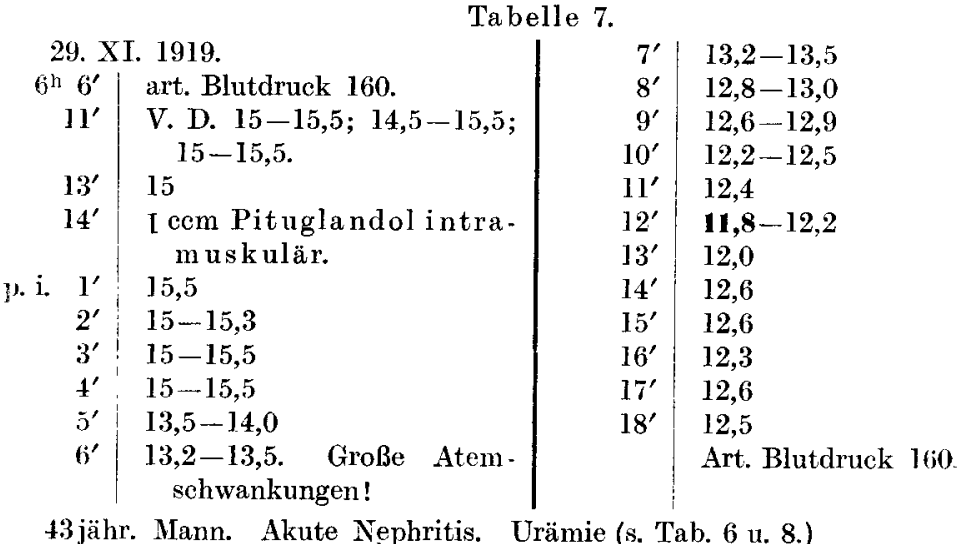

Tabelle 8.

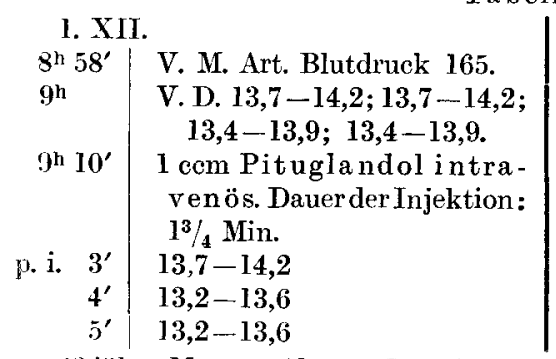

\begin{tabular}{r|l}
$6^{\prime}$ & $13,2-13,6$ \\
$7^{\prime}$ & $128-13,2$ \\
$8^{\prime}$ & $12,5-13,0$ \\
$9^{\prime}$ & 13,2 \\
$10^{\prime}$ & $12,6-13,0$ \\
$11^{\prime}$ & $12,8-13,3$ \\
$12^{\prime}$ & 12,9 \\
$13^{\prime}$ & $12,7-12,1$ \\
$14^{\prime}$ & $12,7-13,1$ \\
& Art. Blutdruck 160.
\end{tabular}

43 jähr. Mann. Akute Nephritis. Urämie (s. Tab. 6 u. 7.) 
Drucksenkung, aber auch kaum eine Drucksteigerung, bewirkte das Pituglandol bei einem Kranken mit totalem Herzblock und Dyspragia intestinalis intermittens arteriosclerotica.

\section{Tabelle 9.}

10. XII. 1919.

\begin{tabular}{|c|c|}
\hline $9^{\text {h }} 15^{\prime}$ & $\begin{array}{l}\text { V. M. Art. Blutdruck } 140 \text {. } \\
\text { V. D. } 4,5 ; 4,2 ; 4,5 ; 4,0 ; 4,2 \text {. }\end{array}$ \\
\hline $9^{\mathrm{h}} 25^{\prime}$ & $\begin{array}{l}\text { V. D. } 4,6 ; 1 \text { ccm Pituglandol } \\
\text { intramuskulär. }\end{array}$ \\
\hline$I^{\prime}$ & 4,8 \\
\hline $2^{\prime}$ & 4,7 \\
\hline $3^{\prime}$ & 4,8 \\
\hline $4^{\prime}$ & 5,1 \\
\hline $5^{\prime}$ & 4,9 \\
\hline $6^{\prime}$ & 4,8 \\
\hline
\end{tabular}

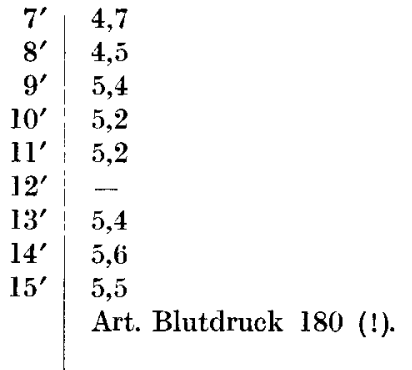

60 jähr. Mann. Herzblock. Arteriosklerose, Dyspragia intestinalis intermittens.

Das Verhalten des arteriellen Blutdrucks war wechselnd, ein sicherer Abfall (um $25 \mathrm{~cm} \mathrm{Hg}$ ) war zweimal (Tab. $10 \mathrm{~b}$ und 11) festzustellen, eine Steigerung nur einmal (Tab. 9).

Tabelle 10 a.

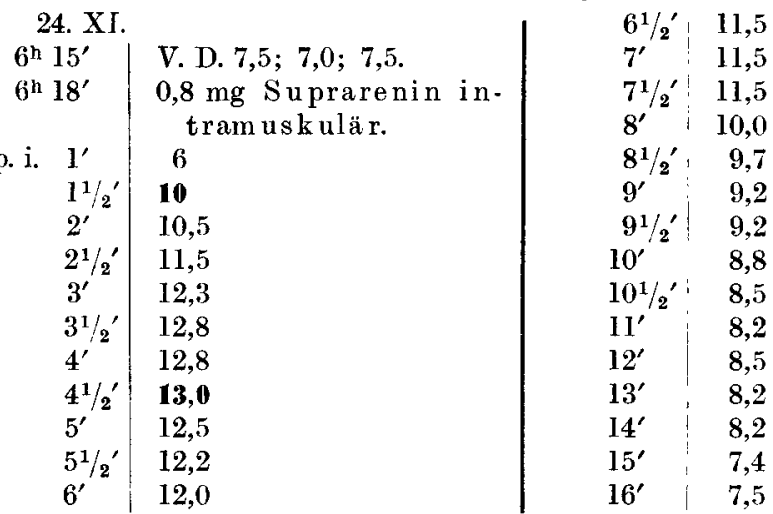

48jähr. Mann. Angina pectoris. Asthma bronchiale (s. Tab. 5b).

Tabelle $10 \mathrm{~b}$.

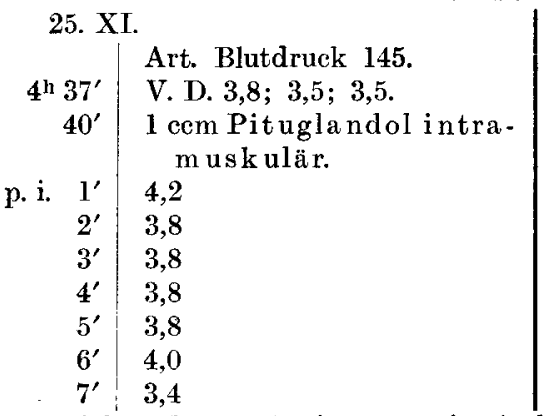

\begin{tabular}{r|l}
$8^{\prime}$ & 3,3 \\
$9^{\prime}$ & 2,5 \\
$10^{\prime}$ & 2,8 \\
$11^{\prime}$ & 2,5 \\
$12^{\prime}$ & 22 \\
$13^{\prime}$ & 2,0 \\
$14^{\prime}$ & 2,0 \\
$15^{\prime}$ & 1,4 \\
$16^{\prime}$ & 1,6 \\
$17^{\prime}$ & 1,4 \\
$5^{h}$ & Art. Blutdruck $120(!)$.
\end{tabular}

48 jähr. Mann. Angina pectoris, Asthma bronchiale. 
Tabelle 11.

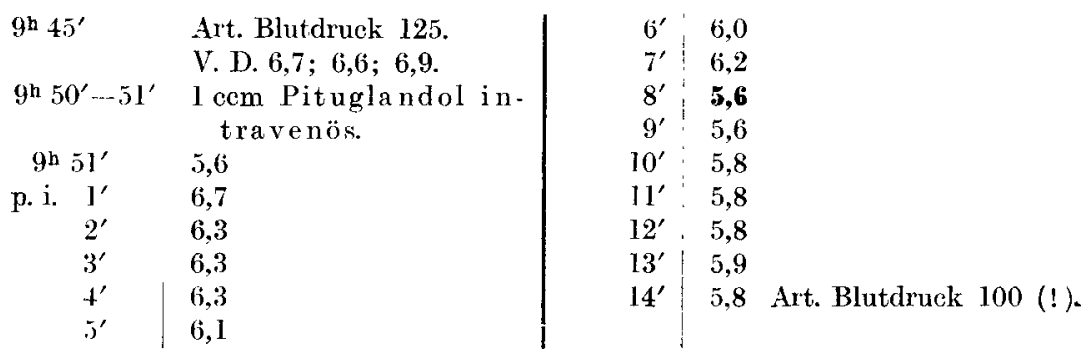

17 jähr. Mann. Verdacht anf Ulcus ventriculi.

Sehr deutlich waren die Unterschiede der Pituglandol-un'd Adrenalinwirkung bei einem Kranken erkennbar. Nach Injektion des Pituglandols sinkt der Venendruck von 3,5-1,4 (s. Tab. 10b), nach Adrenalin erfolgt rascher Anstieg von 7,5-13 ccm! (Tab. 10a).

Will man die vorstehenden Ergebnisse deuten, so lassen sich nicht wie bei der Diskussion der Adrenalinwirkung gesicherte Resultate des Tierversuchs zum Vergleich heranziehen. Jede Erklärung wird mehr oder minder hypothetisch sein müssen.

Zunächst wäre zu erwägen, ob das Absinken des Venendrucks auf eine primäre Erweiterung der Venen bezogen werden kann. Diese Möglichkeit darf wohl als unwahrscheinlich abgelehnt werden, wenn auch an isolierten, ihrer nervösen Verbindungen beraubten Froschgefäßen Hypophysenextrakte entgegen frïheren Feststellungen Dilatation erzeugen sollen [Fröhlich und $\left.\mathrm{Pick}^{\mathbf{1}}\right)$ ]. - Ferner könnte eine Dilatation der Venen des Splanchnicusgebiets der Drucksenkung zugrunde liegen. Für eine solche Auffassung könnten die oben zitierten Versuchsergebnisse von Magnus und Schaefer, Wiggers, Cow herangezogen werden. Im gleichen Sinn verwertbar ist vielleicht.das Fehlen der Druckabnahme bei sicherer Arteriosklerose der Bauchgefäße (s. Tab. 9). Die Vorstellung, daß in diesem Fall das Reservoir der Splanchnicusgefäße sich aus anatomischen Gründen nicht erweitern kann und somit der Abflu $B$ aus der Peripherie erschwert oder unmöglich wird, liegt nahe.

Bei diesen theoretischen Betrachtungen ist absichtlich eine etwaige Einwirkung des Hypophysenextrakts auf das Herz zunächst außer Betracht geblieben. Tatsächlich wird die ganze Frage durch die Beeinflussung der Herztätigkeit sehr viel komplizierter.

Über die Herzwirkung der Hypophysenpräparate beim Menschen weiß man vorläufig wenig. Nach der Injektion von Hinterlappenextrakt sahen Claude, Porak und Routier ${ }^{2}$ ) flüchtige Beschleunigung der Herzaktion, der starke Bradykardie folgte; daneben

1) Archiv f. experim. Pathol. u. Pharmakol. 74, 107. 1913.

2) Compt. rend. de la Soc. de Biol. 14, 205 u. 360. 1912. 
traten Rhythmusstörungen (Vorhof- und Ventrikelextrasystolen) auf. Leschke ${ }^{1}$ ) fand nach Hypophyseninjektion zuweilen kurzdauernde (oft nur wenige Sekunden lange!) Erhöhung von Pulsfrequenz und Blutdruck, dann erst Blutdrucksenkung (s.o.) und häufig starke, vom Sinus ausgehende Arhythmie, in einem Fall Extrasystolie (Pulsus bigeminus).

Bei Gelegenheit plethysmographischer Untersuchungen (vgl. die demnächst in dieser Zeitschrift erscheinende Arbeit) ist mir ebenfalls in einzelnen Fällen eine Beeinflussung der Herzaktion nach der Injektion von Hypophysenextrakten aufgefallen. Unmittelbar nach der Einspritzung kam es zu einer starken Tachykardie mit gleichzeitiger erheblicher Vergrößerung der Pulsamplitude, die als Ausdruck einer Zunahme des Schlagvolumens angesehen werden kann. Eine solche Vergrößerung des Schlagvolumens [die ïbrigens auch im Tierversuch ${ }^{2}$ ) nicht konstant gefunden worden ist] würde jedenfalls durch stärkere Ausschöpfung des venösen Systems den venösen Druck vermindern können [vgl. die diesbezüglichen theoretischen Erörterungen von Moritz und v. Tabora $\left.\left.{ }^{3}\right)\right]$. Es muß 3 aber ausdrücklich betont werden, daß die bisherigen vereinzelten Beobachtungen nur mit allem Vorbehalt zu derartigen Schlüssen verwertet werden dürfen.

Als sicheres Ergebnis der vorstehenden Untersuchungen ist also nur die Tatsache der Herabsetzung des Venendrucks nach Injektion von Pituglandol anzusehen; ob dieser Effekt auf den Venendruck nur durch Beeinflussung der Gefäße oder als Resultante einer Kombination von Gefäß- und Herzwirkungen zu erklären ist, muß zunächst unentschieden bleiben.

1) 1. c.

2) Die älteren Arbeiten sämtlich bei Biedl, Innere Sekretion 3. Aufl. 1916. Neuere Lit. bei Helene Börner, Archiv f. experim. Pathol. u. Pharmakol. r9, 218. 1916, und Herm. Müller, ibid. 81, 238. 1917.

3) Deutsches Archiv f. klin. Med. 98, 475. 1910. 